\title{
ON METHODS OF MEASURING RADIANT EFFICIENCIES.
}

By E. L. Nichols and W. W. Coblentz.

FVER since the publication of the paper of Julius Thomsen ${ }^{1}$ on the Mechanical Equivalent of Light, in which he described the method of separating the luminous from the non-luminous energy of flames by the interposition of a water cell, it has been the general practice of students of artificial illumination to estimate radiant efficiency by some modification of his method.

It was the practice for many years to use instead of water a solution of alum, this substance being supposed to possess more complete opacity for the infra-red than water alone. Melloni is commonly cited as authority. Table II., however, on page 165 of the Thermochrose; in which the transmission of the rays of an Argand burner, by twenty-eight different liquids contained in glass cells is given, shows that according to his measurements such a cell containing a solution of alum transmitted 12 per cent. while the same cell when filled with distilled water allowed I I per cent. only of the radiation to pass.

In 1893 Ernest Nichols, ${ }^{2}$ who studied the transmission spectra of various substances in the infra-red, showed in a much more definite and conclusive manner the almost complete identity with water of the solution of both potassium alum and ammonium alum, as regards their transmitting power. Indeed in the case of solid alum it to the doubtless to the water of crystallization rather than to. the salt that the opacity is to be ascribed.

That neither water nor alum in solution, which may be regarded as identical with the former as regards its transmitting power, fulfills even approximately the conditions necessary to a screen for the separation of the non-luminous radiation has long been known, and it has been customary to make a correction for the imperfect opac-

1 Thomsen, Julius; Poggendorf's Annalen, XXV., page 348.

2 Nichols, E. F., Physical Review, Vol. I., p. I. 
ity of the water cell by means of a cell containing a solution of iodine in carbon bisulphide.

Evidence of the inadequacy of the correction obtained by this method and consequently of the inaccuracy of all our data, for the radiant efficiency of artificial light sources, that have been obtained by the method of the water cell has recently been afforded by Ångström. ${ }^{1}$ He determined the radiant efficiency of the acetylene flame by an ingenious method in which the infra-red radiation is separated from the light-giving rays by the interposition of an opaque screen in the spectrum, after which the light is reassembled and its energy compared with the total energy from the flame. Ångström found for the radiant efficiency by this entirely independent method the value .056 which is a little more than half as great as that obtained by Stewart and Hoxie with a similar flame by the use of the water and iodine cells. ${ }^{2} \mathrm{He}$ finds that all radiant efficiencies hitherto published, with the possible exception of those given by Langley, ${ }^{3}$ who made use of the method of plotting the energy curve for a source of light and of integrating the areas of the luminous and non-luminous portions, are much too large.

A recent investigation by one of the present writers ${ }^{4}$ of the infra-red spectrum of iodine both in the solid form and in solution shows conclusively that the assumption upon which the use of iodine solutions for the purpose of determining the correction which it is necessary to apply to the infra-red radiation transmitted by the water-cell, namely, that iodine solutions which are of sufficient concentration to absorb completely the rays of the visible spectrum are fully transparent in the infra-red, is unwarranted. It is our purpose in the present paper to determine as definitely as possible the degree of inaccuracy involved in the usual method of measuring radiant efficiencies; and with this end in view we have made the following measurements.

Radiant Efficiency by the Method of the Water Cell.

A cylindrical acetylene flame from a single-tipped burner was mounted and the deflection of a Nichols radiometer with rock-salt

\footnotetext{
1 Ångström, Astrophysical Journal, Vol. XV., p. 223, 1902.

2 Nichols, Physical Review, Vol. XI., p. 215, 1900.

${ }^{3}$ Langley, Philosophical Magazine, 30, p. 26, 189o."

${ }^{4}$ Coblentz, W. W., Physical Review, 16, p. 35, 1903.
} 
window was noted when exposed to the direct radiation of this flame; also when a water cell with thin glass walls and containing a layer of distilled water $\mathrm{I} \mathrm{cm}$. in thickness was interposed; and finally when an iodine cell was placed behind the water cell so that the rays had to pass through the two. The iodine cell was of the same dimensions as the water cell. It contained a solution of iodine in carbon bisulphide of such concentration that the filament of an incandescent lamp viewed through the cell was just on the point of extinction. The result of five series of readings was as follows :

TABLE I.

\begin{tabular}{c|c|c}
\hline $\begin{array}{c}\text { Deflections } \\
\text { (Direct). }\end{array}$ & $\begin{array}{c}\text { Deflections } \\
\text { (Through Water Cel1). }\end{array}$ & $\begin{array}{c}\text { Deflections } \\
\text { (Through Both Cells) }\end{array}$ \\
\hline 15.40 & 2.29 & 0.98 \\
15.25 & 2.30 & 1.00 \\
15.35 & 2.29 & 0.99 \\
15.22 & 2.29 & 1.03 \\
15.28 & 2.36 & 1.00 \\
\hline 15.300 (mean) & 2.306 (mean) & 1.000 (mean) \\
\hline
\end{tabular}

The energy transmitted by the water cell according to the above data is $2.306 / 15.300=.1507$.

The proportion of radiation passing both cells as compared with that of passing the water cell alone is $1.00 / 2.306=.4337$.

The radiant efficiency of the flame computed in the usual manner would be $.1507 \times(1-0.4337)=.086$. This value is somewhat lower than that obtained by Stewart \& Hoxie (.105) for a flame of the Naphey type. That the flame of burners of the Naphey type is in general whiter than the flame from the single-tipped burner and presumably therefore is of a higher temperature and of a somewhat higher radiant efficiency, has already been shown by means of a spectrophotometric examination of these flames. ${ }^{1}$

\section{Radiant Efficiency by Integration of the Energy Curve.}

The energy curve of the spectrum of the flame was obtained by using a mirror spectrometer with rock-salt prism, and measuring the intensity of radiation throughout the spectrum by means of the 
Nichols radiometer. This curve of observed intensities was corrected for slit-width variations by means of Paschen's equation:

$$
\alpha f(x)=F(x)-\frac{1}{6} F_{1}(x) .
$$

A further correction was likewise introduced on account of the selective character of the transmission by the rock-salt window which was found to be much less transparent for the shorter than for the longer wave-lengths.

In making these measurements of the spectrum a set of three readings was obtained for each of a series of wave-lengths, one reading for the total intensity of the spectrum at the point in question, one for the radiation transmitted through the water cell and one for the radiation transmitted through the iodine cell; these being placed separately and successively between the flame and the spectrometer slit. The curve of intensities when no cell was interposed is plotted both in the observed and corrected form in Fig. I.

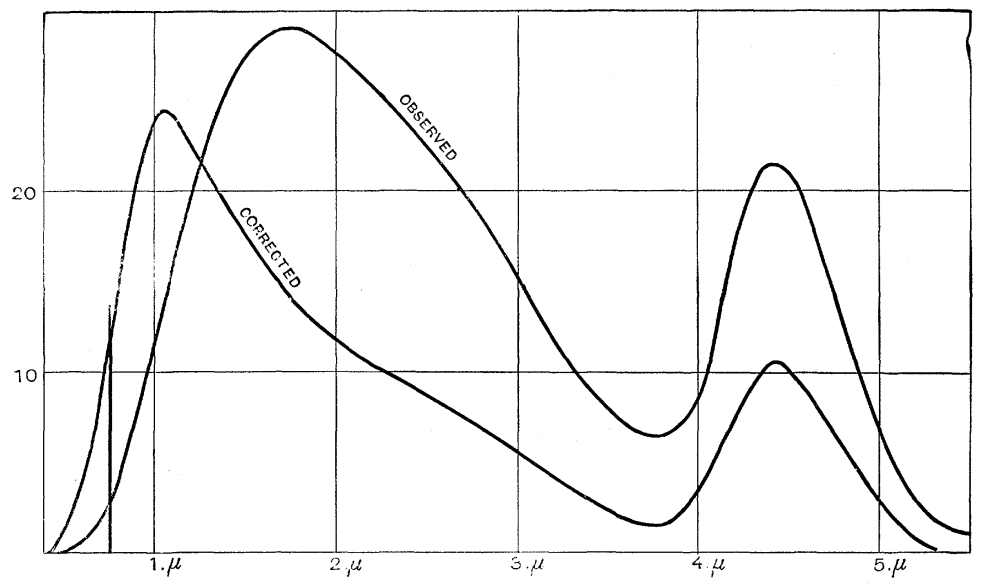

Fig. 1.

In this diagram the area included within the limits of the visible spectrum is separated from the infra-red by the vertical line at $7.6 \mu$. It is evident from a glance at these areas that the value for the radiant efficiency calculated by the method of a water cell is very much too large. The integration of the corrected curve, by means of a planimeter, gave as the ratio of the area of luminous energy to that of total energy, 0.033 . 
Transmission Through Water and Iodine.

The transmission of the water cell and of the iodine cell computed from the readings described above is shown in Fig. 2, in

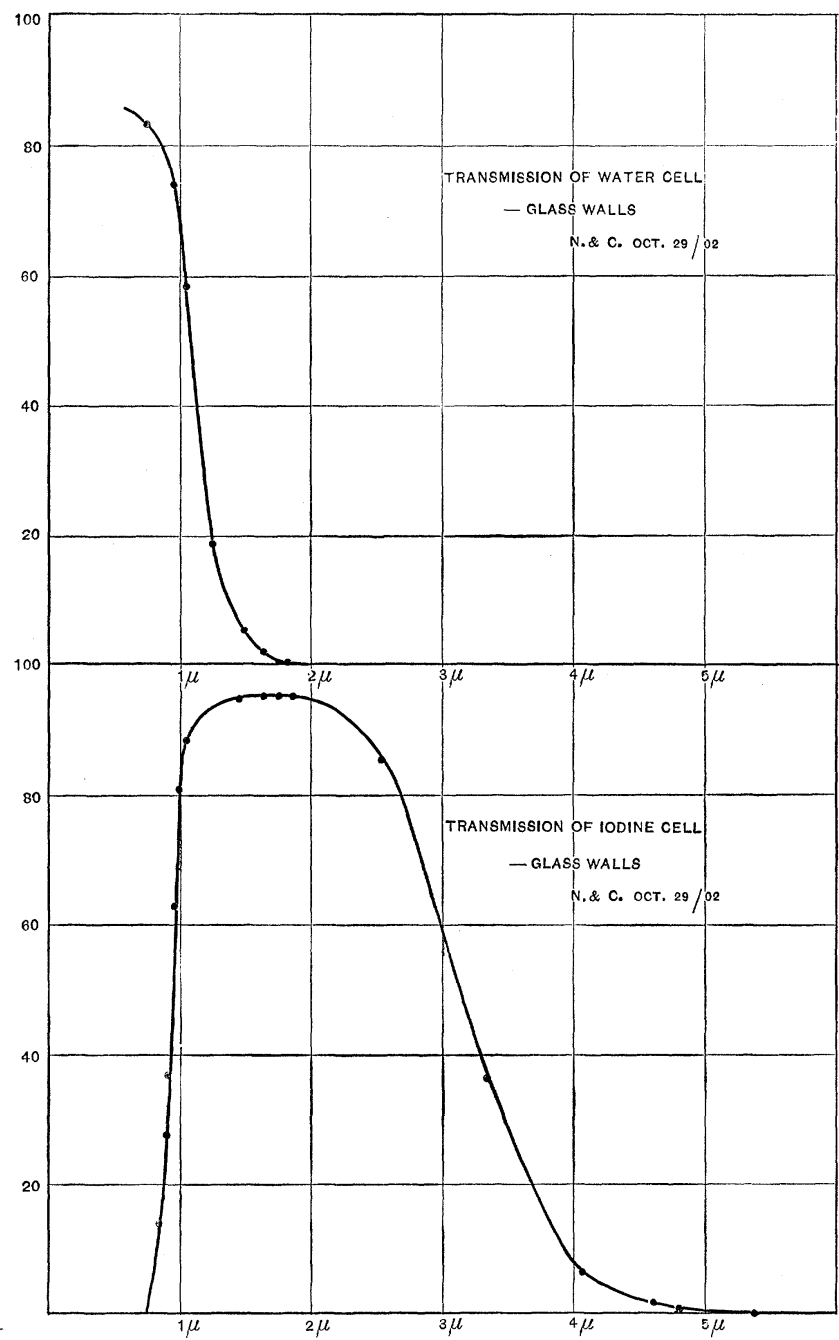

Fig. 2.

which ordinates give the percentage of transmitting power. Complete transparency would be represented by roo, complete opacity by zero. 
It will be seen that the water cell, so far from being opaque throughout the infra-red, transmits 67 per cent. at wave-length i $\mu$ and becomes completely opaque only when wave-length 1.8 is reached. The iodine cell, the function of which in the determination of radiant efficiency, is based upon the assumption that it will transmit whatever infra-red radiation may pass through the water cell, is likewise very far from fulfilling this condition : for although

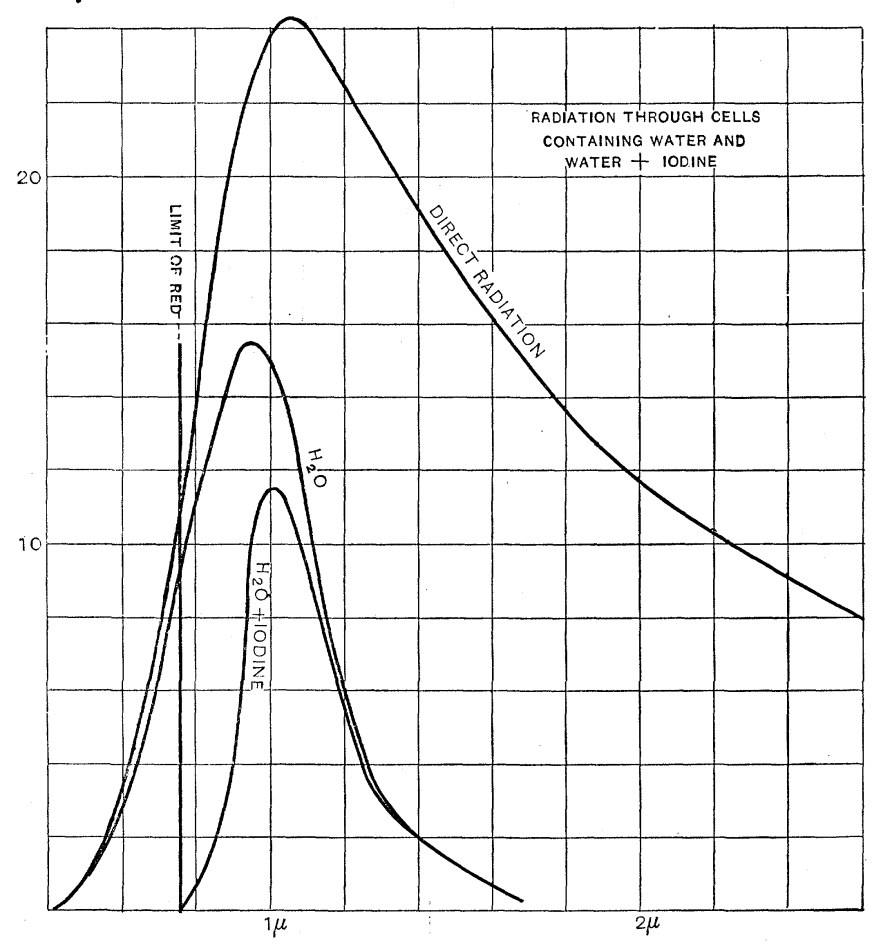

Fig. 3.

the transition from opacity to transparency is very rapid, the solution does not become fully transparent until the region $\mathrm{I} .5 \mu$ is reached.

The precise significance of the data obtained by the water cell method and the complete failure of the iodine cell to serve as a means of correcting for the transparency of water in the infra-red is better shown in Fig. 3, in which a portion of the energy curve for the flame is reproduced. The energy transmitted by the water cell is 
shown by the curve marked $\mathrm{H}_{2} \mathrm{O}$ and that portion of the energy thus transmitted which gets through the iodine cell when the latter is placed behind the water cell is shown by the curve marked $\mathrm{H}_{2} \mathrm{O}+$ iodine. These curves indicate clearly the very large error involved in the use of the iodine cell and lead us to the rejection of it altogether in the study of radiant efficiencies.

An integration of the area of luminous energy and of the total energy enclosed in the curve marked $\mathrm{H}_{2} \mathrm{O}$ shows that of the energy transmitted by the water cell only about one sixth, more accurately 0.174, falls within the visible spectrum. A comparison of the area measuring the luminous energy transmitted by the water cell with the area representing the total luminous energy of the flame shows that the losses of luminous energy by reflection and transmission in passing through this cell amount to I 3 per cent. In order to check this ratio, the precise determination of which with the radiometer is somewhat precarious on account of the small deflections obtained in the visible spectrum, two similar acetylene flames were mounted at the end of a photometer bar and the ratio of their intensities was determined. The water cell was then placed in front of one of them and the measurement repeated. The transparency of the water cell thus measured was found to be 0.87 , a value which is in agreement with that obtained by the integration of the curves. The computation of the radiant efficiency of the flame from the data obtained by the integration of these curves gives us . $1507 \times$ $.174 / .87=.030 ;$ a value somewhat smaller than that obtained from the integration of the energy curve itself.

Both of these values for the radiant efficiency are surprisingly low. The method of integrating the energy curve for the determination of the ratio of luminous energy to the total area, while it appears at first sight to be the most direct and therefore likely to give the best value, is unfortunately subject to considerable errors arising from the difficulty of making the proper correction for variations of slit-width within the luminous area. The radiometer readings for this portion of the spectrum are always small and the change in the slit-width, measured in wave-lengths, undergoes its most rapid changes as we pass over from the visible to the invisible spectrum. Uncertainties concerning the trend of the dispersion 
curve of the prism which are apparently insignificant affect the ordinates of the corrected energy curve in this region very notably. Fortunately we have the means of checking the accuracy of the results in this case by comparing the ratio of the area of the curve representing the total transmission of the water cell to that of the total area of the energy curve with the corresponding ratio obtained from the measurement of the deflections due to the unrestricted and undispersed radiation from the flame as compared with the deflections produced when the water cell was interposed in the path of the rays. The ratio of the areas by integration is 1.528 while that obtained by the latter method is, as we have already seen. 1.507. The close agreement of these ratios is such as to give confidence in the substantial accuracy of the two methods.

An explanation of the low value of the efficiency of this flame is to be found in the peculiar character of its energy curve. It will be noted that the emission band at $4.4 \mu$ is very large. The area of the region lying between $4.0 \mu$ and $4.9 \mu$ is in point of fact .192 of the total area of the curve. Integration of the corresponding area of a curve for a cylindrical flame measured by G. W. Stewart and corrected by Paschen's equation shows for the corresponding region an area of .0948 as compared with the total area of that curve. The radiant efficiency of Stewart's flame as determined by integration of the luminous and total areas gives .040.

The existence of a very powerful emission band at wave-length 44.4 is characteristic of flames of the Bunsen type; as may be seen from the curve given by Stewart ${ }^{1}$ in his paper on the spectrum of the acetylene flame. The very large emission band at this wavelength, in the case of the flame upon which the measurements described in the present paper were made, probably indicates that the air ducts in the burner were too large as compared with the orifice through which the acetylene gas reached the tip.

A reduction in the supply of acetylene from partial clogging of the orifice would tend to convert the flame into the Bunsen type and to reduce its radiant efficiency. Measurements of the spectrum of the flame on this particular burner made on different days, show that the intensity of the band varies through a wide range.

IStewart, Physical Review, Vol 13, p. 272, I9or. 
The ratio of the deflection at the top of this band to that at the maximum of the energy curve on April 26 was .40. On October 29, the day of the readings from which the curve shown in Fig. I was plotted, it was .76. On November 8 it was .50; on December I 3 it was .59 .

Measurements with the water cell at a time when this ratio had a value of .50 gave for the ratio of the transmitted to the direct radiation .200 as against the .150 of Table I. The radiant efficiency on that day, assuming the ratio for luminous to total transmission through the water cell not to be appreciably changed, was $.174 \times .20 / .87=.040$. This value is identical with that obtained by integration of the curve for Stewart's flame.

We have in these observations without doubt an explanation of the principal difficulty of using the acetylene flame as a primary standard in photometry. The gas orifice in all existing types of the acetylene burner is very small and the partial clogging of this orifice would serve to modify to a serious extent the quality and the total brightness of the flame.

\section{Conclusions.}

The observations recorded in this paper suffice to show the very large correction which must be applied to the ratio between the energy transmitted by a water cell and the total radiation if one is to obtain a correct value for the radiant efficiency of any source of light; also the futility of the method of ascertaining this correction by means of the iodine cell. It is obvious that the factor by which this ratio must be multiplied will vary with the radiant efficiency, and while the nature of this variation is perhaps comparatively simple in the case of sources in which the light is derived from incandescent carbon the value of the correction for other sources can only be ascertained when we have definite information concerning the character of the infra-red spectrum. Were we to apply, to take an extreme case, the method of the water cell to the radiation from sodium vapor at the temperature of the electric arc we should have to do with an infra red spectrum nearly all of the energy of which lies, according to the curves published by Snow, ${ }^{1}$ at wave-lengths less than I. $4 \mu$. The infra-red spectrum of this vapor consists chiefly of

${ }^{1}$ Snow, Physical Review, Vol. I., p 95. 
two strong lines or pairs of lines at $.82 \mu$ and I.1 $3 \mu$. There is, it is true, a group of lines or bands lying between I.7 $\mu$ and I.9 $\mu$ but the energy of these is insignificant. What may lie beyond $2 \mu$ we do not know. A very large part of the energy of such a source of light would presumably be transmitted by the water cell. In the case of the Arons mercury lamp according to measurements reported elsewhere by Coblentz and Geer ${ }^{1}$ we have in the infra-red a group of lines which lie within the region of transparency of the water cell and a second group to which the cell would be entirely opaque. In the study of such sources of light determinations of the energy transmitted by the water cell are without significance. Results obtained by exploring the entire spectrum and plotting the energy curve would, on account of the difficulty of making a satisfactory correction for the variations of slit width, likewise be of doubtful value. To determine the radiant efficiency of such sources we must have recourse to a method based upon the principles used by Ångström in his recent paper or we must find some screen which will be opaque to the entire infra-red, and the transmitting power of which for the visible spectrum can be determined photometrically.

Physical Laboratory of Cornell University, December 24, 1902.

${ }^{1}$ Coblentz and Geer, Physical Review, Vol. XVI., p. 279. 